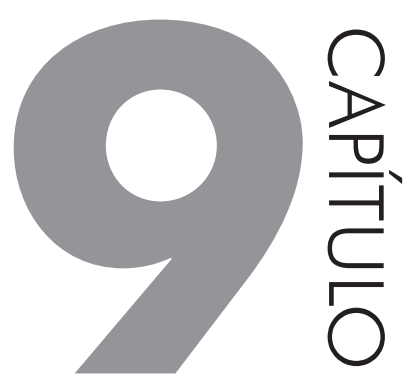

\title{
MOBILE LEARNING NO BRASIL: UM ESTUDO EXPLORATÓRIO DA LITERATURA
}

\section{ROBERTA MOCELIN, PATRICIA JANTSCH FIUZA, ROBSON RODRIGUES LEMOS}

\section{INTRODUÇÃO}

Desde a pré-história a vida do homem é constantemente alterada por novas tecnologias que ele mesmo descobre ou cria. Começando pela invenção da escrita, passando pela descoberta do fogo e da criação de ferramentas com pedra, chegando ao nascimento de tecnologias digitais, cada fato deste foi uma tecnologia que revolucionou o comportamento humano. Desde o advento da internet, e a popularização de computadores pessoais vive-se uma era digital, onde as tecnologias surgem em ritmo quase frenético, e verdadeiramente estão transformando as relações que os indivíduos têm, seja com os dispositivos eletrônicos, seja entre eles mesmos. (Kenski, 2012; Veen \& Vrakking, 2009; Fiuza \& Gonçalves, 2017).

$\mathrm{Na}$ última década, uma nova categoria de dispositivos digitais chegou ao mercado, e ganhou um espaço importante na vida das pessoas: os dispositivos móveis. Com a capacidade de serem transportados para qualquer lugar, sem a necessidade de estarem constantemente plugados à energia, os dispositivos móveis se tornaram uma ferramenta quase que indispensável ao homem, permitindo que ele possa estar conectado à internet e fazer praticamente tudo que um computador tradicional faz. Os desenvolvedores criaram os mais variados programas para este dispositivo (conhecidos como aplicativos móveis), e cada vez mais servi- 
ços foram disponibilizados para serem realizados através destes equipamentos. Hoje, é possível encontrar uma infinidade de marcas e modelos de tablets e Smartphones, que agradam as diferentes classes sociais, estando presente tanto em países subdesenvolvidos, quanto nos desenvolvidos.

Junto com a popularização dos dispositivos wireless (sem-fio), nasce uma geração de crianças que desde os primórdios de sua criação estão expostas a este tipo de tecnologia. Dentro de suas casas os pais possuem Smartphones, tablets e notebooks e não hesitam em incentivar seus filhos a utilizar tais aparelhos. (Prensky, 2001; Veen \& Vrakking, 2009).

Como já dito, os dispositivos móveis proporcionam uma liberdade de tempo e espaço, e quando conectados à internet ampliam os canais de informação que o usuário tem acesso. Tal característica proporciona um viés pedagógico até então desconsiderado por muitas pessoas, que encaram tais dispositivos apenas como opção de entretenimento ou comunicação.

A popularização dos dispositivos móveis deu origem a uma nova modalidade de aprendizagem que se baseia no uso deste tipo de equipamento. O mobile learning, ou aprendizagem móvel, têm surgido como uma alternativa para aproveitar os benefícios que estes dispositivos apresentam. No entanto, a aplicação deste conceito não é nada fácil, e muitos pais e professores ainda têm dificuldades para enxergar o potencial pedagógico presente nos dispositivos digitais.

Ainda que este tema seja relativamente novo, é possível encontrar muitos exemplos de sucesso em diferentes países do mundo (Shuler, 2009). E no Brasil, o que tem se falado sobre este tema? Há iniciativas em Mobile Learning brasileiras? Buscando conhecer mais esta realidade e responder as perguntas que surgem neste novo cenário que se organizou este trabalho.

\section{O QUE CARACTERIZA O MOBILE LEARNING}

Mobile Learning diz respeito à mobilidade de um aprendiz. Este conceito defende que a aprendizagem deve ser independente de um espaço físico para acontecer. Kukulska-Hulme (2005) afirma que o aprendizado fora da sala de aula depende apenas da motivação para tanto, ao identificar uma oportunidade. O diferencial é a possibilidade de se valer de pequenos dispositivos, capazes de caberem nos bolsos, tais como Smartphones e tablets. Notebooks também são considerados, devido a sua característica portátil (Kukulska-Hulme, 2005).

Em 2013, a Unesco publicou um documento chamado "Diretrizes de Políticas para o Aprendizado Móvel”, onde defende o uso de dispositivos móveis no ensino. Neste documento, o termo aprendizagem móvel está definido como: "A aprendizagem móvel envolve o uso de tecnologias móveis, isoladamente ou em 
combinação com outras Tecnologias da Informação e Comunicação (TIC's), a fim de permitir a aprendizagem a qualquer hora e em qualquer lugar”. (Unesco, 2013, p. 8)

O texto ainda esclarece que a aprendizagem móvel pode ocorrer de diferentes formas, seja usando os dispositivos móveis para acessar ou criar conteúdo educativo, ou para conectar-se com outras pessoas e trocar informações. Além disso, ressalta-se que esta modalidade de ensino “(...) abrange esforços em apoio a metas educacionais amplas, como a administração eficaz de sistemas escolares e a melhor comunicação entre escolas e famílias". (Unesco, 2013, p. 8)

Sabe-se que os dispositivos móveis desempenham um papel importante na vida de adultos e crianças, e agora há um movimento para estudar e identificar que papel estes dispositivos podem exercer no aprendizado das pessoas. No entanto, ainda se esbarra na falta de investimentos e iniciativas em relação ao aprendizado móvel, que na maioria das vezes é posto em prática por "um professor inovador, um pesquisador apaixonado, um desenvolvedor arriscado ou um pai inspirador" (Shuler, 2009, p. 15). Os esforços em Mobile Learning são muito pontuais e estão espalhados pelo mundo, alguns apresentando sucesso. Enquanto estas iniciativas ajudam a disseminar o potencial da aprendizagem móvel, é necessário que o meio acadêmico, indústria e governo combinem esforços para ampliar ainda mais o alcance deste método de ensino. (Shuler, 2009)

\section{MOBILE LEARNING NO BRASIL}

Para investigar o Mobile Learning no contexto educacional brasileiro, optou-se por pesquisar em bases de dados a temática da aprendizagem móvel através de uma metodologia chamada Revisão Sistemática da Literatura (RSL). Esta metodologia se caracteriza como uma técnica replicável, pois segue um processo padrão para sua execução. Além disso, é considerada transparente, pois o pesquisador deixa claro os passos seguidos na pesquisa, o que confirma o caráter replicável deste método. Uma RSL revela como o tema investigado vem sendo tratado nas publicações desde a sua primeira incidência nas bases de dados, e é uma importante ferramenta para elaborar uma base de conhecimento sobre o assunto, uma vez que se pode observar o quanto e o que já foi estudado e identificar qual a necessidade de demais estudos sobre o tema. (Fiuza \& Mocelin, 2016)

Para Freire (2013, p. 30), “a RSL é um processo de levantamento de dados onde são exigidas revisões rigorosas de publicações acadêmicas" com o intuito de identificar evidências sobre determinado assunto. Esta metodologia de pesquisa é do tipo exploratório que busca identificar, selecionar e avaliar de maneira crítica 
os estudos existentes sobre o tema. Freire (2013, p. 30) ainda destaca que "por eliminar a possibilidade de o pesquisador dirigir aleatoriamente a seleção de dados, a RSL evita o seu viés”. Portanto, esta característica evita que a pesquisa seja conduzida de maneira tendenciosa.

É importante ressaltar que a intenção de uma RSL não é produzir novos resultados ou encontrar respostas diretas a determinada questão, mas sim encontrar o que já foi e o que precisa ser estudado sobre o assunto abordado na investigação realizada através da revisão sistemática. (Tranfield, Denyer \& Smart, apud Brinner \& Denyer, 2012)

\section{EXECUÇÃO DA RSL}

Para a realização da RSL necessária neste trabalho foi utilizada uma ferramenta específica para a execução deste tipo de pesquisa chamada StArt. Esta ferramenta é de uso livre, foi desenvolvida pelo Laboratório de Pesquisa em Engenharia de Software (Lapes) da Universidade Federal de São Carlos (UFSCAR) e está disponível para download na página web do laboratório citado. O StArt apresenta todos os recursos necessários para viabilizar a elaboração de uma RSL de maneira organizada e coerente e a partir da realização do download desta ferramenta e sua devida instalação em um computador, os primeiros passos da RSL foram dados.

Primeiramente, foi elaborado o Protocolo de Pesquisa. Esta etapa é de suma importância estratégica na realização da revisão, uma vez que neste estágio o pesquisador determina aonde quer chegar e de que maneira chegará. A elaboração do protocolo de pesquisa deve conter:

- Pergunta de revisão: pode ser descrita como o ponto de partida para a busca de estudos que estejam relacionados à esta questão, com o objetivo de identificar o cenário em que a pergunta está sendo realizada. É necessário que ela seja elaborada com clareza e com as devidas delimitações ao tema, para que todos possam compreender o que está sendo buscado. (Fiuza \& Mocelin, 2016)

A pergunta de pesquisa elaborada para guiar a RSL apresentada neste trabalho é a seguinte: Como o Mobile Learning tem sido aplicado nas instituições de ensino brasileiras?

Esta questão de pesquisa deixa claro que o intuito da revisão é identificar iniciativas de Mobile Learning nas instituições de ensino do Brasil, bem como descobrir que tipo de resultados tais experiências obtiveram.

- Critérios de inclusão e exclusão: são critérios escolhidos pelo pesquisador para determinar quais publicações serão classificadas como válidas para 
serem utilizadas no estudo. Podem ser períodos de tempo, metodologia empregada, objetivo, tipo de estudo, entre outros. (Freire, 2013)

As tabelas a seguir demonstram os critérios de inclusão e exclusão utilizados.

Tabela 1 Critérios de inclusão.

\begin{tabular}{|l|l|}
\hline \multicolumn{1}{|c|}{ CRITÉRIO DE INCLUSÃo } & \multicolumn{1}{|c|}{ JUSTIFICATIVA } \\
\hline Estudo realizado no Brasil. & Esta investigação se limita ao Brasil. \\
\hline Publicação do tipo estudo de caso. & Por apresentar relatos de experiência com M-learning. \\
\hline Estudos com exemplos de aprendizagem móvel. & Para descobrir iniciativas de M-learning. \\
\hline Estudos em inglês ou português. & Línguas relevantes para o tema e região investigada. \\
\hline
\end{tabular}

Fonte: Autores.

Tabela 2 Critérios de exclusão.

\begin{tabular}{|l|l|}
\hline \multicolumn{1}{|c|}{ CRITÉRIO DE EXCLUSÃo } & \multicolumn{1}{c|}{ JUSTIFICATIVA } \\
\hline Estudo realizado no exterior. & O estudo de concentra no Brasil. \\
\hline Publicação em outras línguas. & Não há relevância para este estudo. \\
\hline Ausência de exemplos práticos em M-learning. & $\begin{array}{l}\text { O foco deste estudo é encontrar iniciativas práticas em apren- } \\
\text { dizagem móvel. }\end{array}$ \\
\hline Publicações tipo livro ou capítulo de livro. & Por serem de difícil acesso. \\
\hline Publicações que fujam do tema M-learning. & Por não serem pertinentes ao tema da revisão. \\
\hline Monografias, dissertações e teses. & $\begin{array}{l}\text { Por se tratarem de trabalhos extensos e mais detalhados, } \\
\text { aumentando o tempo necessário para a realização da fase de } \\
\text { extração dos dados. }\end{array}$ \\
\hline
\end{tabular}

Fonte: Autores.

- Estratégia de busca: definir como e onde os estudos serão buscados.

Para esta revisão foi escolhido um método de busca por palavras chaves nas bases de dados Scopus e Google Academic. Esta última foi escolhida especialmente por trazer maior volume de publicações em português. Para efetuar a busca em cada base de dados, uma combinação string de palavras-chave é montada para que o sistema faça uma busca destas palavras no título, resumo e palavras-chave de cada publicação. 
Tabela 3 Busca de publicações.

\begin{tabular}{|l|l|c|}
\hline \multicolumn{1}{|c|}{ BASE DE DADOS } & \multicolumn{1}{|c|}{ STRING } & $\begin{array}{c}\text { QUANTIDADE } \\
\text { DE ESTUDOS } \\
\text { RETORNADOS }\end{array}$ \\
\hline Scopus & $\begin{array}{l}\text { (mobile AND devices) AND (mobile AND learning) AND (limit-to } \\
\text { (language, "Portuguese")) }\end{array}$ & 50 \\
\hline Google Academic & $\begin{array}{l}\text { "Tecnologias digitais" AND "dispositivos móveis" AND "aprendi- } \\
\text { zagem móvel" AND "mobile learning" AND "escolas" AND "Brasil" } \\
\text { AND "estudo de caso" }\end{array}$ & 72 \\
\hline Subtotal & & 122 \\
\hline
\end{tabular}

Fonte: Autores.

É importante ressaltar que as strings de busca acabaram sendo diferentes, pois os bancos de dados utilizados usam maneiras distintas de filtragem dos resultados. Portanto, mais filtros tiveram de ser aplicados no Google Academic para que o resultado fosse mais coerente com a pergunta de pesquisa que guiou esta revisão.

Freire (2013) recomenda que, além da pesquisa por palavras-chave, uma busca dirigida ao tema também seja realizada para complementar a revisão. Sendo assim, optou-se por buscar pelos autores de dissertações e teses retornados na pesquisa nos bancos de dados com a expectativa de encontrar outras publicações que retratassem os seus trabalhos. Esta etapa adicionou outros seis artigos, totalizando 128 publicações encontradas nesta primeira etapa de RSL.

- Seleção dos estudos: nesta fase, os resumos dos 128 artigos obtidos na pesquisa foram analisados a fim de identificar o contexto, metodologia e assunto de cada publicação. Desta forma foi possível separar os estudos que aparentavam ter mais relevância de acordo com a pergunta de pesquisa que guiou a revisão. Para realizar esta separação os critérios de inclusão e exclusão previamente definidos foram aplicados com base nas informações presente nos resumos. Ao final deste processo, 71 publicações demonstravam afinidade com o tema principal e se enquadravam nos critérios de inclusão, portanto foram selecionadas para que seu texto completo fosse lido e seu conteúdo analisado mais precisamente.

- Extração e análise de dados: nesta etapa da RSL os 71 estudos escolhidos na fase de seleção foram lidos por completo, com o intuito de 
compreender e analisar mais profundamente cada publicação, uma vez que na fase anterior apenas os resumos haviam sido estudados. Para isso, elaborou-se uma espécie de formulário, onde foram listados alguns itens que se esperava encontrar no texto para que este estivesse dentro do contexto idealizado de acordo com a pergunta de pesquisa. A construção deste formulário é disponibilizada pela ferramenta StArt, utilizada para a realização da revisão sistemática. Os itens inclusos foram: tipo de instituição de ensino, conteúdo trabalhado com Mobile Learning, tipo de dispositivo utilizado, tempo de duração do experimento, classificação do resultado obtido e espaço para demais comentários sobre o estudo.

Nesta fase também foi possível reaplicar os critérios de inclusão e exclusão, visto que a partir da leitura completa dos textos identificou-se informações que não estavam presentes no resumo. Esta também foi uma maneira de excluir alguns estudos que apresentaram características que se enquadravam nos critérios de exclusão.

A leitura completa dos textos juntamente com o preenchimento do formulário e a reaplicação dos critérios citados resultou na exclusão de mais 54 estudos, deixando um montante final de 17 estudos. Estes 17 estudos escolhidos apresentaram relevância com o tema, bem como tinham as características necessárias para satisfazer a pergunta de pesquisa. Os resultados finais da revisão sistemática de literatura realizada neste trabalho serão apresentados a seguir.

\section{RESULTADOS DA REVISÃO SISTEMÁTICA DE LITERATURA}

Ao concluir os passos de seleção dos estudos e extração dos dados neles contidos, executou-se uma meta-análise, definido por Luiz (2002, p. 409) como "um método quantitativo que permite combinar os resultados de estudos realizados de forma independente (...) e sintetizar as suas conclusões ou mesmo extrair uma nova conclusão". Freire (2013, p. 38) complementa afirmando que a meta-análise "promove a combinação estatística de pelo menos dois estudos, para produzir uma estimativa única”.

Através desta meta-análise alguns gráficos foram elaborados demonstrando aspectos importantes relacionando os 17 estudos finais. A Figura 1 apresenta o primeiro gráfico cujo tema é o ano das publicações. Os estudos foram publicados entre 2011 e 2017, tendo maior volume de publicações nos anos de 2015 e 2016. Isto sinaliza que o interesse em aprendizagem móvel está crescendo, e considerando que o presente estudo foi conduzido ainda no primeiro semestre de 2017 espera-se que ao longo deste ano a quantidade de estudos publicados nesta temática 
continue a crescer. Este é um sinal de que pesquisadores e instituições estão atentas às mudanças necessárias nos métodos de ensino, e estão buscando soluções para este cenário.

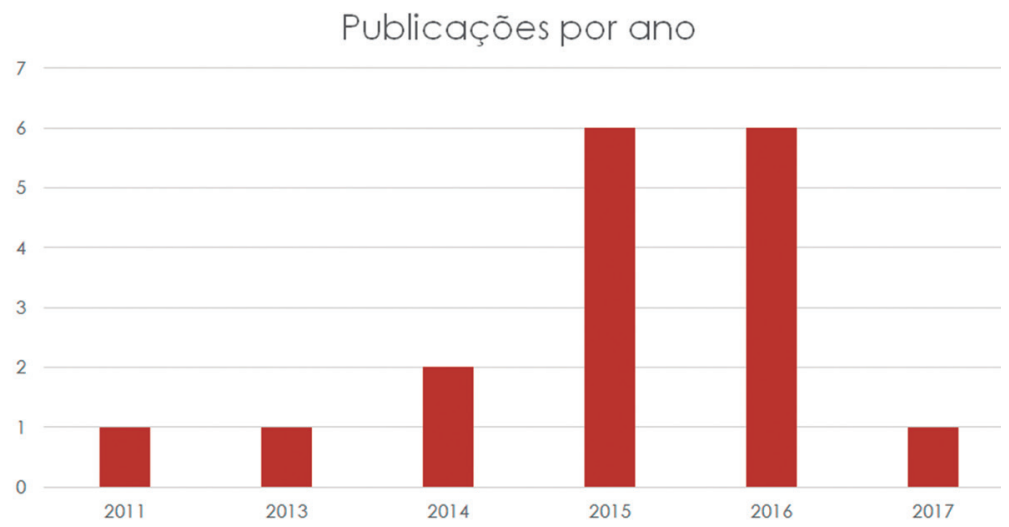

Figura 1 Publicações por ano.

Fonte: Autores.

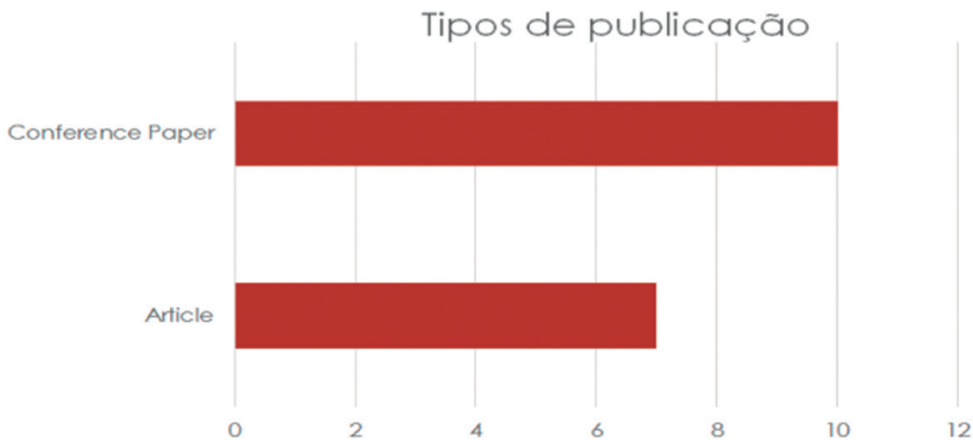

Figura 2 Tipos de publicação.

Fonte: Autores.

A Figura 2 mostra o gráfico que separa por quantidades os tipos de publicações admitidos na RSL conduzida, sendo estes artigos e conference papers, que se refere à publicações aceitas em eventos. Do total de 17 estudos, 10 foram do tipo conference paper e 7 do tipo artigo.

A Figura 3 apresenta as disciplinas trabalhadas nas experiências de Mobile Learning retratadas nas publicações. A nomenclatura dos conteúdos foi elaborada conforme sua descrição nos próprios estudos. Como houveram experiências 
desde o ensino fundamental até o superior, tentou-se agregar da melhor forma possível os diferentes tipos de conteúdo em suas respectivas áreas. A parte descrita como "outros" engloba conteúdos de disciplinas específicas de ensino superior de diferentes cursos, dificultando elaborar a relação com uma área mais abrangente para cada assunto.

Sendo assim, pode-se identificar que a maioria dos estudos trabalhou matemática ou ciências através das mais variadas abordagens se valendo de dispositivos digitais. Notou-se também um esforço no ensino superior para utilizar técnicas de aprendizagem móvel em conteúdos diferenciados, específicos aos seus respectivos cursos de graduação.

\section{Disciplinas abordadas com M-Learning}

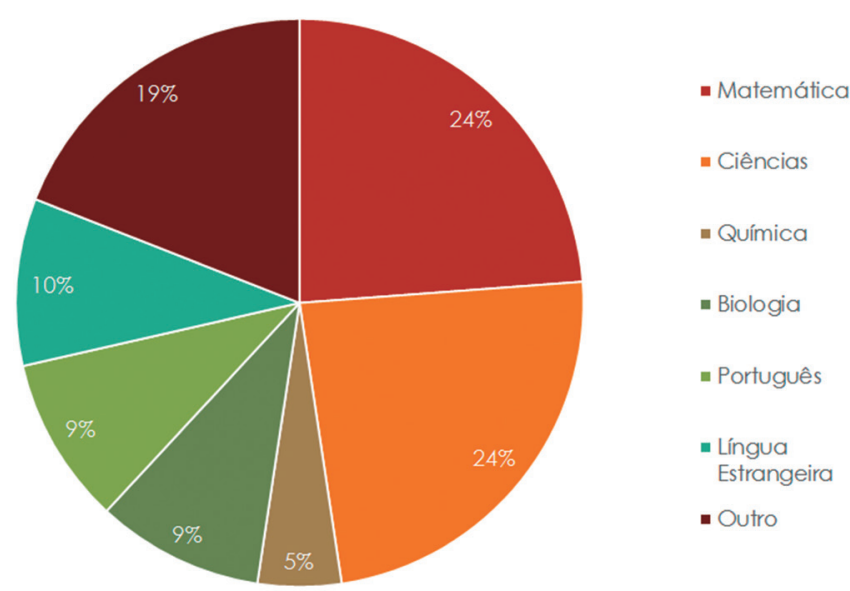

Figura 3 Assuntos trabalhados com aprendizagem móvel.

Fonte: Autores.

A Figura 4 ilustra o gráfico que demonstra os tipos de instituições de ensino que receberam as iniciativas de aprendizagem móvel conduzidas pelos pesquisadores. Optou-se por separar em escolas públicas ou privadas (até o ensino médio ou técnico) e universidades em geral. É importante destacar que alguns estudos foram conduzidos em mais de um tipo de instituição, enquanto outros omitiram o tipo de instituição estudada. Neste último caso não há como presumir esta informação, portanto não consta no gráfico a totalidade dos estudos.

Observou-se que a maioria dos estudos de casos foram aplicados em escolas públicas mesmo que estas apresentem menor potencial tecnológico quando comparadas às instituições privadas. Isto demonstra que é possível incluir dispositivos digitais e aprendizagem móvel nas salas de aulas públicas, desde que haja um pla- 
nejamento para contornar os possíveis problemas ocasionados pela falta de ferramentas tecnológicas nestas situações.

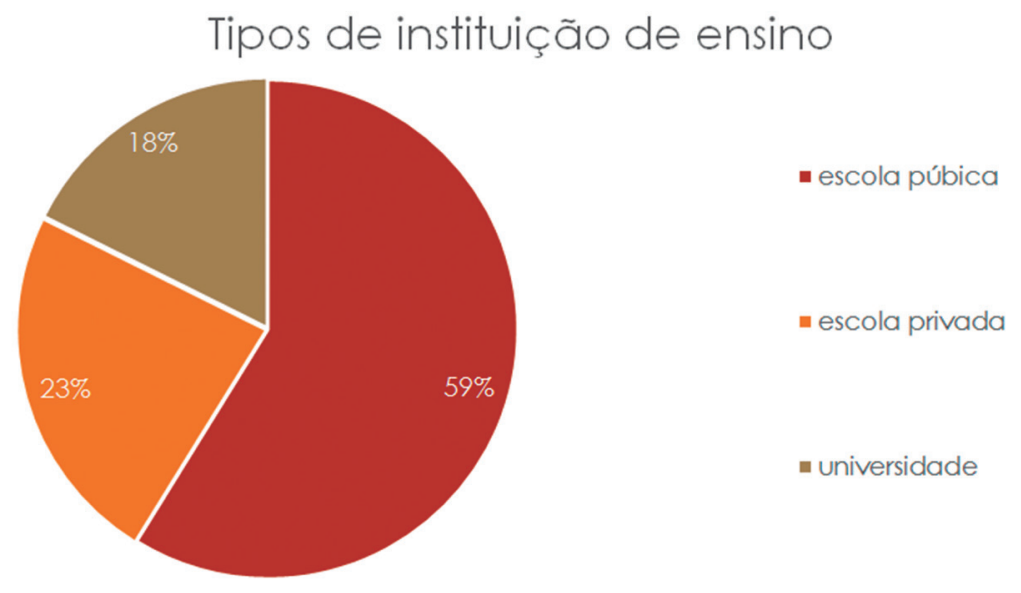

Figura 4 Tipos de instituição de ensino.

Fonte: Autores.

A Figura 5 representa o gráfico que diz respeito aos tipos de dispositivos utilizados nas experiências descritas nos estudos. Foram listados Smartphones, tablets, computador portátil (notebooks ou netbooks) e outros (no caso de algum tipo de dispositivo diferenciado). O Smartphone foi o dispositivo mais utilizado, reforçando o potencial pedagógico deste aparelho que está cada vez mais presente no cotidiano do estudante. O segundo dispositivo mais utilizado foi o tablet que é muito semelhante ao Smartphone quanto às suas especificações e usabilidade.

Apesar destes dois dispositivos mais usados não serem ferramentas desenvolvidas com cunho pedagógico, elas oferecem muitos recursos que podem ser adaptados para esta finalidade. O número de aplicativos educacionais desenvolvidos para estes dispositivos tem aumentado nos últimos anos, agregando valor pedagógico a estes aparelhos. Além disso, uma aula pode ser planejada usando ferramentas que não sejam inicialmente pedagógicas e mesmo assim estar se valendo de mobile learning. O uso da câmera fotográfica ou uso do browser nativo do aparelho para buscas na internet, por exemplo, pode alimentar o processo de aprendizagem de acordo com a atividade proposta.

Feitos estes levantamentos quantitativos, uma análise descritiva foi realizada para demonstrar o que e como os estudos desenvolvem o tema da pesquisa. Eles foram combinados qualitativamente e serão apresentados nos quadros a seguir. 


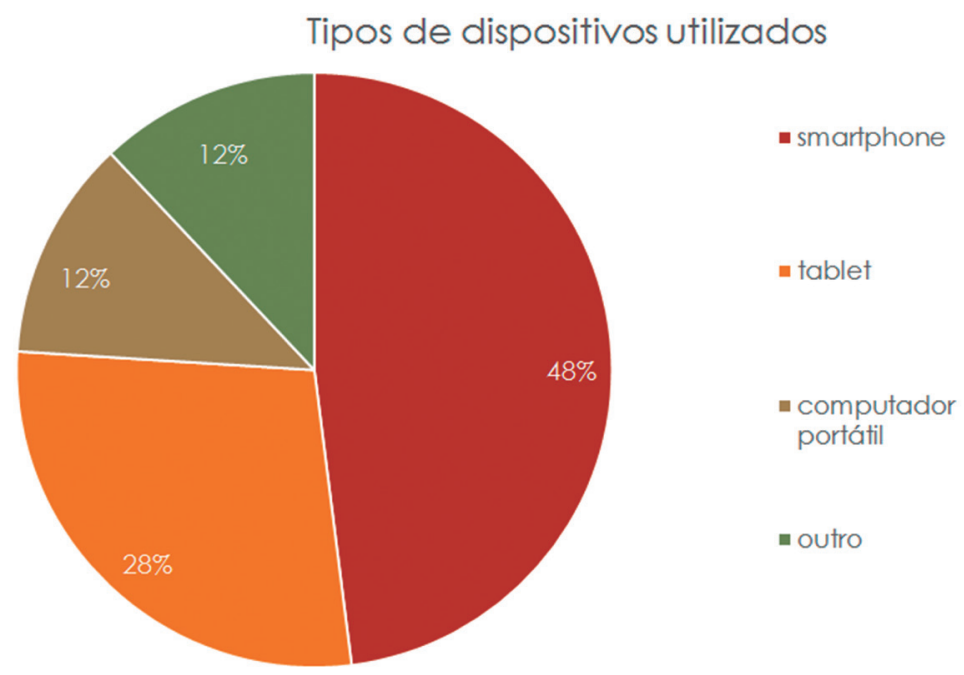

Figura 5 Dispositivos utilizados nas experiências de M-learning.

Fonte: Autores.

Tabela 4 Mobile Learning na matemática.

\begin{tabular}{|l|l|l|l|}
\hline \multicolumn{1}{|c|}{ AUTORES } & \multicolumn{1}{|c|}{ TítULO } & DISPOSITIVo & \multicolumn{1}{c|}{ EXPERIÊNCIA } \\
\hline $\begin{array}{l}\text { Pereira, Silva e } \\
\text { Piconez (2016) }\end{array}$ & $\begin{array}{l}\text { Mathematics experiences: Peda- } \\
\text { gogical implications with the use } \\
\text { of digital games, m-learning and } \\
\text { social networks. }\end{array}$ & Smartphones & $\begin{array}{l}\text { Uso de jogos digitais não pedagó- } \\
\text { gicos para trabalhar o raciocínio } \\
\text { matemático de alunos do 80 e 90 } \\
\text { ano. }\end{array}$ \\
\hline $\begin{array}{l}\text { Silva e } \\
\text { Schimiguel } \\
\text { (2017) }\end{array}$ & $\begin{array}{l}\text { Mobile Learning e sua influên- } \\
\text { cia para o desenvolvimento de } \\
\text { pessoas. }\end{array}$ & Tablets & $\begin{array}{l}\text { Jogo educacional MathTimer com } \\
\text { alunos do ensino médio. }\end{array}$ \\
\hline $\begin{array}{l}\text { Homann, } \\
\text { Barbosa e } \\
\text { Martins (2016) }\end{array}$ & $\begin{array}{l}\text { Aprendizagem baseada em jogos } \\
\text { digitais educativos para o ensino } \\
\text { da matemática. }\end{array}$ & Tablets & $\begin{array}{l}\text { Jogo Navegáticas para reforçar o } \\
\text { conteúdo de equações nos anos } \\
\text { finais do ensino fundamental. }\end{array}$ \\
\hline $\begin{array}{l}\text { lahnke, Botelho } \\
\text { e Ferreira (2016) }\end{array}$ & $\begin{array}{l}\text { Colmeias: Um estudo de caso na } \\
\text { matemática. }\end{array}$ & Smartphones & $\begin{array}{l}\text { Uso de redes sociais para reali- } \\
\text { zação de atividades colaborativas } \\
\text { sobre geometria plana. }\end{array}$ \\
\hline $\begin{array}{l}\text { Batista, Behar, } \\
\text { Passerino e } \\
\text { Mamari (2011) }\end{array}$ & $\begin{array}{l}\text { Celular como ferramenta de } \\
\text { apoio pedagógico ao cálculo. }\end{array}$ & Smartphones & $\begin{array}{l}\text { Acesso a AVA's com atividades } \\
\text { sobre cálculo I através de disposi- } \\
\text { tivos móveis. }\end{array}$ \\
\hline
\end{tabular}


Tabela 5 Mobile Learning nas ciências.

\begin{tabular}{|c|c|c|c|}
\hline AUTORES & TÍTULO & DISPOSITIVO & EXPERIÊNCIA \\
\hline $\begin{array}{l}\text { Hitzschky, Brito, } \\
\text { Arruda, Lima, } \\
\text { Melo e Castro } \\
\text { Filho (2016) }\end{array}$ & $\begin{array}{l}\text { Práticas educativas com o uso de dis- } \\
\text { positivos móveis em aulas de campo: } \\
\text { Aprendizagem para além dos muros } \\
\text { da escola. }\end{array}$ & $\begin{array}{l}\text { Smartphones } \\
\text { tablets, outros }\end{array}$ & $\begin{array}{l}\text { Grupo em rede social para com- } \\
\text { partilhar conhecimento e uso } \\
\text { de dispositivos móveis em saí- } \\
\text { da de campo. }\end{array}$ \\
\hline $\begin{array}{l}\text { Almeida, Lopes } \\
\text { e Lopes (2015) }\end{array}$ & $\begin{array}{l}\text { Sequências didáticas eletrônicas no } \\
\text { ensino do corpo humano: comparan- } \\
\text { do o rendimento do ensino tradicional } \\
\text { com o ensino utilizando ferramentas } \\
\text { tecnológicas. }\end{array}$ & Tablet & $\begin{array}{l}\text { Jogos e atividades relacionadas } \\
\text { com o corpo humano com alu- } \\
\text { nos do } 8^{\circ} \text { ano. }\end{array}$ \\
\hline $\begin{array}{l}\text { Ribeiro, San- } \\
\text { tana, Nobre, } \\
\text { Sondermann e } \\
\text { Vieira (2016) }\end{array}$ & $\begin{array}{l}\text { O uso de tecnologias móveis no ensi- } \\
\text { no de ciências: uma experiência sobre } \\
\text { o estudo dos ecossistemas costeiros } \\
\text { da mata atlântica sul capixaba. }\end{array}$ & Smartphones & $\begin{array}{l}\text { Uso do app Map of Life e dispo- } \\
\text { sitivos móveis para registro de } \\
\text { saída de campo para estudar } \\
\text { ecossistemas. }\end{array}$ \\
\hline $\begin{array}{l}\text { Almeida, Costa, } \\
\text { Nascimento, } \\
\text { Lopes e Lopes } \\
\text { (2015) }\end{array}$ & $\begin{array}{l}\text { Trabalhando a agenda } 21 \text { na escola: } \\
\text { Utilizando tablets como ferramenta de } \\
\text { ensino. }\end{array}$ & Tablets & $\begin{array}{l}\text { Coleta de dados e desenvolvi- } \\
\text { mento de atividades com o ta- } \\
\text { blet sobre sustentabilidade com } \\
\text { alunos do } 8^{\circ} \text { ano. }\end{array}$ \\
\hline $\begin{array}{l}\text { Lima, Lima } \\
\text { Neto e Castro } \\
\text { Filho (2015) }\end{array}$ & $\begin{array}{l}\text { Aprendizagem móvel no ensino fun- } \\
\text { damental }\end{array}$ & $\begin{array}{l}\text { Smartphones e } \\
\text { netbooks }\end{array}$ & $\begin{array}{l}\text { Atividades colaborativas sobre } \\
\text { sustentabilidade através do } \\
\text { google drive, e troca de infor- } \\
\text { mações em grupo do whatsapp. }\end{array}$ \\
\hline $\begin{array}{l}\text { Silva e Schimi- } \\
\text { guel (2017) }\end{array}$ & $\begin{array}{l}\text { Mobile Learning e sua influência para o } \\
\text { desenvolvimento de pessoas. }\end{array}$ & Smartphones & $\begin{array}{l}\text { Pesquisa sobre tópicos de quími- } \\
\text { ca com alunos do ensino médio. }\end{array}$ \\
\hline
\end{tabular}

Tabela 6 Mobile Learning em língua portuguesa.

\begin{tabular}{|l|l|l|l|}
\hline \multicolumn{1}{|c|}{ AUTORES } & \multicolumn{1}{|c|}{ TítUlo } & DISPOSITIVo & \multicolumn{1}{c|}{ EXPERIÊNCIA } \\
\hline $\begin{array}{l}\text { Conforto e } \\
\text { Vieira (2015) }\end{array}$ & $\begin{array}{l}\text { Smartphone na escola: da discus- } \\
\text { são disciplinar para a pedagógica }\end{array}$ & Smartphones & $\begin{array}{l}\text { Uso de AVA e QR codes para busca de } \\
\text { informação e melhorar a construção de } \\
\text { argumentos para redações de alunos do } \\
\text { ensino médio. }\end{array}$ \\
\hline $\begin{array}{l}\text { Oliveira } \\
\text { e Castro } \\
\text { (2016) }\end{array}$ & $\begin{array}{l}\text { Rede social como recurso didáti- } \\
\text { co-pedagógico: Possibilidade de }\end{array}$ & Smartphones & $\begin{array}{l}\text { Compartilhamento de informações, dú- } \\
\text { vidas e materiais sobre português para } \\
\text { prova do Enem entre professores e alu- } \\
\text { nos em grupo do Whatsapp. }\end{array}$ \\
\hline
\end{tabular}


Tabela 7 Mobile Learning em língua estrangeira.

\begin{tabular}{|l|l|l|l|}
\hline \multicolumn{1}{|c|}{ AUTORES } & \multicolumn{1}{|c|}{ TíTULO } & DISPOSITIVO & \multicolumn{1}{c|}{ EXPERIÊNCIA } \\
\hline $\begin{array}{l}\text { Marciano, Oli- } \\
\text { veira, Menezes } \\
\text { e Miranda } \\
\text { (2015) }\end{array}$ & $\begin{array}{l}\text { Katakana star samurai: A mobile tool } \\
\text { to support learning of a basic japane- } \\
\text { se alphabet. }\end{array}$ & $\begin{array}{l}\text { Smartphonese } \\
\text { tablets }\end{array}$ & $\begin{array}{l}\text { Proposta de jogo para ensino de } \\
\text { um tipo de alfabeto japonês. }\end{array}$ \\
\hline $\begin{array}{l}\text { Silva e Schimi- } \\
\text { guel (2017) }\end{array}$ & $\begin{array}{l}\text { Mobile Learning sua influência para } \\
\text { o desenvolvimento de pessoas. }\end{array}$ & Smartphones & $\begin{array}{l}\text { Atividades propostas com uso do } \\
\text { celular para alunos do ensino médio. }\end{array}$ \\
\hline
\end{tabular}

Tabela 8 Mobile Learning em outras disciplinas.

\begin{tabular}{|l|l|l|l|}
\hline \multicolumn{1}{|c|}{ AUTORES } & \multicolumn{1}{|c|}{ TíTULO } & DISPOSITIVo & \multicolumn{1}{c|}{ EXPERIÊNCIA } \\
\hline $\begin{array}{l}\text { Duarte Filho, } \\
\text { Goncalves \& } \\
\text { Pizetta (2014) }\end{array}$ & $\begin{array}{l}\text { Experimental analysis of the efi- } \\
\text { ciency of application e-mages in me- } \\
\text { dical imaging visualization. }\end{array}$ & Tablets & $\begin{array}{l}\text { Teste de um aplicativo para análise } \\
\text { de imagens médicas com estudan- } \\
\text { tes e profissionais de medicina }\end{array}$ \\
\hline $\begin{array}{l}\text { Sonego e Behar } \\
\text { (2015) }\end{array}$ & $\begin{array}{l}\text { M-learning: Reflexões e perspecti- } \\
\text { vas com o uso de aplicativos edu- } \\
\text { cacionais. }\end{array}$ & $\begin{array}{l}\text { Smartphone, } \\
\text { tablets, outros }\end{array}$ & $\begin{array}{l}\text { Alunos do curso de pedagogia de- } \\
\text { senvolveram aplicativos com temas } \\
\text { apresentados na disciplina de Mídia, } \\
\text { Tecnologias Digitais e Educação. }\end{array}$ \\
\hline Lima, Bassani e \\
Barbosa (2014) & $\begin{array}{l}\text { Aprender com mobilidade: utiliza- } \\
\text { e comunicação moveis e sem o } \\
\text { como potencializadoras da intera- } \\
\text { ção em processos educativos. }\end{array}$ & $\begin{array}{l}\text { Smartphone, } \\
\text { tablets, outros }\end{array}$ & $\begin{array}{l}\text { Acompanhamento de alunos em } \\
\text { exercício domiciliar através de blo- } \\
\text { gs, redes sociais, chats, etc. Ativi- } \\
\text { dades cooperativas para manter o } \\
\text { contexto escolar. }\end{array}$ \\
\hline Gomes (2013) & $\begin{array}{l}\text { Potencialidades e limites pedagó- } \\
\text { gicos na utilização dos dispositivos } \\
\text { móveis na educação de jovens e } \\
\text { adultos. }\end{array}$ & Smartphone & $\begin{array}{l}\text { Alunos do EJA produziram material } \\
\text { sobre uma visita ao observatório } \\
\text { para o conteúdo de astronomia. }\end{array}$ \\
\hline
\end{tabular}

\section{CONSIDERAÇÕES FINAIS}

A busca por publicações brasileira com o tema "Mobile Learning" foi bem-sucedida, visto que muitas das publicações retornadas traziam de fato uma discussão sobre o tema, e foi possível encontrar uma boa quantidade de estudos de caso sobre iniciativas em aprendizagem móvel no Brasil. Observou-se que este tema tem sido escolhido por muitos mestrandos e doutorandos para serem desenvolvidos em suas dissertações e teses, pois o número destes tipos de trabalhos retornados na pesquisa foi grande. 
As publicações encontradas são recentes, tendo a mais antiga destas, apenas 6 anos. Considerando que o acesso à internet através de dispositivos móveis vem crescendo, e que segundo dados da $11^{a}$ pesquisa TIC Domicílios teve um aumento de $9 \%$ no caso do acesso através do celular entre 2014 e 2016, é esperado que o interesse pelo tema deste trabalho também aumente (Portal Brasil, 2016). Cada ano que passa surgem mais possibilidades de inclusão da tecnologia digital no ensino e à medida que a necessidade desta inclusão também aumenta, espera-se que mais pesquisas e experiências sejam efetivadas pelos pesquisadores brasileiros na busca por soluções de tecnologia da informação na educação do país.

No entanto, é importante ressaltar um detalhe em comum a todos os estudos analisados nesta revisão: nenhum deles apresentou indícios de que houve continuidade. As experiências são muitas, nos diversos assuntos e disciplinas desde o ensino fundamental ao superior. Apesar de serem, como já foi dito, iniciativas recentes, ao que parece, foram todas experiências isoladas, que aconteceram pelo tempo determinado da pesquisa, mas em nenhuma publicação declarou-se que o esforço para implantação de aprendizagem móvel era uma constante nos respectivos ambientes de estudo, ou que seriam mantidas como um projeto a longo prazo. Ainda que todos os casos apresentem sucesso e tenham validado pontos importantes de mobile learning, receia-se que o esforço terminou com o estudo relatado e as aulas voltaram a ser como tradicionalmente eram feitas.

Muitas são as justificativas para esta falta de continuidade de iniciativas de aprendizagem móvel nas escolas brasileiras. Falta de equipamentos adequados nas escolas, despreparo dos educadores, e a própria legislação municipal ou estadual são exemplos de barreiras ao trabalho continuado em M-learning. Na maioria dos casos, por exemplo, as escolas não dispunham de dispositivos para uso dos alunos. Em alguns, os professores tiveram de ser capacitados pelos pesquisadores. Realmente, a aprendizagem móvel requer muito estudo, investigação e planejamento para ser desenvolvida, e alguns profissionais da área da educação não estão dispostos a pisar neste terreno ainda pouco desbravado para dar um caráter mais atual aos seus métodos de ensino.

Outro ponto em comum a todos os estudos foi a satisfação dos alunos em usar a tecnologia como parte das tarefas de aula. Os alunos se sentiam motivados e se engajavam melhor nas atividades quando estas estavam relacionadas com o uso de dispositivos móveis.

Mesmo que a mobilidade permeie as ações cotidianas nos mais diferenciados contextos, no que se refere à educação ainda é preciso subir muitos degraus para atingir o patamar em que o avanço tecnológico se encontra no cotidiano geral da população. A educação precisa acelerar seu processo de atualização, e incorporar de vez o uso da tecnologia digital como apoio no ensino. O professor precisa visualizar nos dispositivos digitais um aliado, e não um inimigo que apenas disper- 
sa a atenção do aluno. Ele precisa deixar de ser o centro da aula, e se tornar o mediador do conhecimento, dando espaço para novas técnicas, metodologias e possibilidades no ensino apoiados na tecnologia.

A realização da RSL deixou claro que existe o interesse em tornar a aprendizagem móvel uma realidade, e muitos esforços para isso têm sido despendidos pelos pesquisadores brasileiros. Os benefícios do uso consciente, planejado e mediado de dispositivos móveis na educação ficou evidente com a revisão, e serve de incentivo para o surgimento de mais iniciativas neste campo. Mesmo que muitas dificuldades sejam encontradas neste caminho, é possível perceber um movimento crescente de apoio a esta causa, e espera-se que nos próximos anos seja possível encontrar iniciativas de Mobile Learning no Brasil que estejam aplicadas de forma constante e eficiente, servindo de modelo para a ampliação da aprendizagem móvel a nível nacional e internacional.

\section{REFERÊNCIAS}

Almeida, C. M. M. de et al. (2015). Trabalhando a agenda 21 na escola: utilizando tablets como ferramenta de ensino. In: II Encontro de Ciências em Educação para a Sustentabilidade. Ulbra. Acesso em: 10 out. 2017, <https://www.researchgate.net/profile/ Paulo_Campos_Lopes/publication/283733236_TRABALHANDO_A_AGENDA_21_ NA_ESCOLA_UTILIZANDO_TABLETS_COMO_FERRAMENTA_DE_ENSINO/ links/56463eb108ae9f9c13e7474e.pdf>.

Batista, S. C. F., Behar, P. A., Passerino, L. M. \& Mamari, A. B. (2011). Sequências didáticas eletrônicas no ensino do corpo humano: comparando o rendimento do ensino tradicional com o ensino utilizando ferramentas tecnológicas. Acta Scientiae, v. 17, n. 2. Acesso em: 10 out. 2017, do <http://www.periodicos.ulbra.br/index.php/acta/article/view/1562/1209>.

Batista, S. C. F., Behar, P. A., Passerino, L. M. \& Mamari, A. B. (2011). Celular como ferramenta de apoio pedagógico ao cálculo. RENOTE, v. 9, n. 1. Recuperado em 26 outubro, 2017, do <http://seer.ufrgs.br/index.php/renote/article/view/21990/12757>.

Brinner, R. B. \& Denyer, D. (2012). Systematic review and evidence synthesis as a practice and scholarship tool. In: Rousseau, D. M. (Ed.). Handbook of evidence-based management: companies, classrooms, and research. New York: Oxford University Press, p. 328-374.

Conforto, D. \& Vieira, M. C. (2015). Smartphone na escola: Da discussão disciplinar para a pedagógica. Latin American Journal of Computing Faculty of Systems Engineering National Polytechnic School, v. 2, n. 3. Acesso em: 10 out. 2017, do <http://lajc.epn. edu.ec/index.php/LAJC/article/view/95/56>.

Duarte Filho, N. F., Gonçalves, C. F. \& Pizetta, D. C. (2014). Experimental analysis of the eficiency of application e-mages in medical imaging visualization. In: IEEE. Information Systems and Technologies (CISTI), 9 ${ }^{\text {th }}$ Iberian Conference on. [S.l.], p. 1-6. Acesso em: 10 out. 2017, do <http://ieeexplore.ieee.org/document/6876925/>. 
Fiuza, P. J. Tecnologias interativas na educação. (2015). In: Malacarne, V. (Ed.). Educação, tecnologias de informação e comunicação e outros olhares. Curitiba, PR: CRV.

Fiuza, P. \& Mocelin, R. (2016). Systematic review of literature: the contributions to the learning process by digital technologies and pedagogical architectures. In: Rocha, Á., Correia, A. M., Adeli, H., Reis, L. P., Mendonça Teixeira, M. (Eds.). New Advances in Information Systems and Technologies. [S.1.]: Springer.

Fiuza, P. J. \& Gonçalves, B. B. S. (2017). Uso de softwares no contexto da pesquisa científica. In: Patricia Jantsch Fiuza; Robson Rodrigues Lemos. (Org.). Inovação em educação: perspectivas do uso das tecnologias interativas. 1ed.Jundiaí/SP: Paco Editorial, v. 1, p. 61-89.

Freire, P. d. S. (2013). Aumente a qualidade e a quantidade de suas publicações científicas. Curitiba: PR: CRV.

Gomes, F. A. R. (2013). Potencialidades e limites pedagógicos na utilização dos dispositivos móveis na educação de jovens e adultos. In: Anais do V Simpósio hipertexto e tecnologia na educação. Acesso em: 10 out. 2017, do <http://nehte.com.br/simposio/anais/ Anais-Hipertexto-2013/POTENCIALIDADES\% 20 E \% 20 LIMITES\% 20 PEDAG\%C3\%93GICOS\%20NA\%20UTILIZA\%C3\%87\%C3\%830\%20DOS\%20 DISPOSITIVOS\%20M\%C3\%93VEIS\%20NA\%20EDUCA $\%$ C3\% 87\%C3\% 83 O $\% 20$ DE\%20JOVENS\%20E\%20ADULTOS.pdf>.

Hitzschky, R. A., Brito, M. A. F. de, Arruda, J. S., Lima, C. A., Melo, M. B. O. de \& Castro Filho, J. A. de. (2016). Práticas educativas com o uso de dispositivos móveis em aulas de campo: aprendizagem para além dos muros da escola. In: Nunes, I. D. \& Maia, L. (Ed.). Anais do Congresso regional de tecnologia na educação. p. 210-219. Acesso em: 10 out. 2017, do <http://ceur-ws.org/Vol-1667/CtrlE_2016_AC_paper_40.pdf>.

Hoffmann, L. F., Barbosa, D. N. F. \& Martins, R. L. (2016). Aprendizagem baseada em jogos digitais educativos para o ensino da matemática. In: Anais do XV Seminário Internacional de Educação. Acesso em: 10 out. 2017, do <https://www.feevale.br/Comum/ midias/fa97183f-74dd-4a51-938b-c960d12e0c2a/Aprendizagem\%20baseada $\% 20$ em $\% 20$ jogos $\% 20$ digitais $\% 20$ educativos $\% 20$ para $\% 20$ o $\% 20$ ensino $\% 20 \mathrm{da} \% 20$ matem $\%$ C3\%A1tica.pdf $>$.

Iahnke, S. L. P., Botelho, S. S. da C. \& Ferreira, A. L. A. (2016). Colmeias: Um estudo de caso na matemática. RENOTE, v. 14, n. 1. Acesso em: 10 out. 2017. <http://seer. ufrgs.br/index.php/renote/article/view/67383/38474.

Kenski, V. M. (2012). Educação e Tecnologias: o novo ritmo da informação. [S.1.]: Campinas, SP: Papiros.

Kukuska-Hulme, A. \& Traxler, J. (2005). Mobile learning: a handbook for educators and trainers. Open and Flexible Learning Series. London, UK: Routledge.

Lima, C. C. de, Bassani, P. B. S. \& Barbosa, D. N. F. (2014). Aprender com mobilidade: utilização das tecnologias da informação e comunicação móveis e sem fio como potencializadoras da interação em processos educativos. RENOTE, v. 12, n. 1. Acesso em: 10 out. 2017. <http://seer.ufrgs.br/index.php/renote/article/view/49828/31186>.

Lima, M. S. S., Lima Neto, C. S. \& Castro Filho, J. A. de. (2015). Aprendizagem móvel no ensino fundamental. In: Anais dos Workshops do Congresso Brasileiro de Informática na Educação. [S.1.: s.n.]. v. 4, n. 1, p. 825-833. Acesso em: 10 out. 2017. <http:// www.br-ie.org/pub/index.php/wcbie/article/view/6125/4293>. 
Luiz, A. J. B. (2002). Meta-análise: definição, aplicações e sinergia com dados espaciais. Cadernos de Ciência \& Tecnologia, v. 19, n. 3, p. 407-428. Acesso em: 10 out. 2017. $<$ https://seer.sct.embrapa.br/index.php/cct/article/view/8814/4955>.

Marciano, J. N., Oliveira, J. B., Menezes, B. C. \& Miranda, L. C. (2015). Katakana star samurai: A mobile tool to support learning of a basic japanese alphabet. In: IEEE. Computing Conference (CLEI), 2015 Latin American. [S.1.]. p. 1-8. Acesso em: 10 out. 2017. <http:// ieeexplore.ieee.org/document/7359973/>.

Oliveira, T. F. de S. \& Castro, A. B. B. de. (2016). Rede social como recurso didático-pedagógico: Possibilidade de uso na aprendizagem de língua portuguesa. In: SIED: EnPED-Simpósio Internacional de Educação a Distância e Encontro de Pesquisadores em Educação a Distância. Acesso em: 10 out. 2017. <http://www.sied-enped2016.ead.ufscar.br/ojs/index.php/2016/article/view/1578/644>.

Pereira, A. B. C., Silva, F. S. C. da \& Piconez, S. C. B. (2016). Mathematics experiences: Pedagogical implications with the use of digital games, m-learning and social networks. In: IEEE. Learning Objects and Technology (LACLO), Latin American Conference. p. 1-9. Acesso em: 10 out. 2017. <http://ieeexplore.ieee.org/document/7751757/>.

Portal Brasil. (2016). Pesquisa revela que mais de 100 milhões de brasileiros acessam a internet. Acesso em: 10 out. 2017. <http://www.brasil.gov.br/ciencia-e-tecnologia/2016/09/pesquisarevela-que-mais-de-100-milhoes-de-brasileiros-acessam-a-internet>.

Prensky, M. (2001). Digital natives, digital immigrants part 1 . On the horizon, MCB UP Ltd., v. 9, n. 5, p. 1-6.

Ribeiro, G., Santana, R., Nobre, I., Sondermann, D., \& Vieira, L. (2016). O uso de tecnologias móveis no ensino de ciências: uma experiência sobre o estudo dos ecossistemas costeiros da mata atlântica sul capixaba. Revista Ibero-Americana de Estudos em Educação, v. 11, n. 4, p. 2234-2244, 2016. Acesso em: 10 out. 2017. <http://seer.fclar.unesp. br/iberoamericana/article/view/9122/6056>.

Shuler, C. (2009). Pockets of potential: using mobile technologies to promote children's learning. Joan Ganz Cooney Center at Sesame Workshop, New York.

Silva, B. de O. \& Schimiguel, J. (2017). Mobile Learning e sua influência para desenvolvimento de pessoas. Revista Atlante: Cuadernos de Educacion y Desarrollo. Acesso em: 10 out. 2017. <http://www.eumed.net/rev/atlante/2017/03/aprendizado.html>. <http:// hdl.handle.net/20.500.11763/atlante1703aprendizado $>$.

Sonego, A. H. S. \& Behar, P. A. (2015). M-learning: reflexões e perspectivas com o uso de aplicativos educacionais. In: XX Congresso Internacional de Informática Educativa (TISE). p. 521-526. Acesso em: 10 out. 2017.<http://www.tise.cl/volumen11/TISE2015/521526.pdf $>$.

Tajra, S. F. (2008). Informática na educação: novas ferramentas pedagógicas para o professor na atualidade. [S.1.]: Erica.

Unesco. (2013). Diretrizes de políticas da Unesco para aprendizagem móvel. Acesso em: 10 out. 2017. <http://unesdoc.unesco.org/images/0022/002277/227770por.pdf>.

Veen, W. \& Vrakking, B. (2009). Homo zappiens: educando na era digital. Porto Alegre: Artmed Editora. 
